\title{
The Eschar of Scrub Typhus
}

Sir,

A 9-yr-old boy presented with twelve days of highgrade intermittent fever with hepatosplenomegaly and a $0.5 \times 0.5 \mathrm{~cm}$ eschar over upper anterior abdominal wall (Fig. 1). Eschar preceded the fever with itch on the site and a papulovesicular lesion. This ruptured to form a dark brown to black crust over 2 days. Scrub typhus Elisa for IgM was positive. With oral doxycyclline his itch disappeared in $12 \mathrm{hrs}$ and fever subsided in $36 \mathrm{hrs}$. On follow-up after 2 wk the eschar had resolved leaving a small scar.

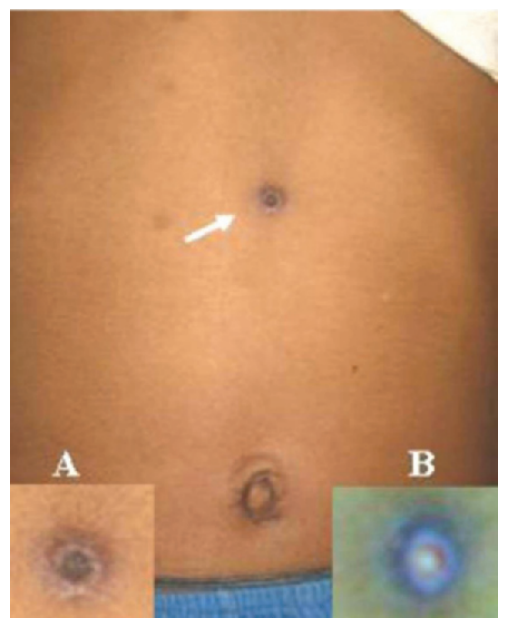

Fig. 1. Typical eschar in upper anterior abdominal wall. (A) Cigarette burn like erythematous halo surrounding a dark necrotic center. (B) Day 10 of therapy: a healing eschar with a punched out center and a black halo.
Scrub typhus or tsutsugamushi disease is a miteborne rickettsiosis encountered globally. As mite bites are painless, patients are unaware even with eschar and/or fever. The infection begins as a red induration, within $48 \mathrm{hrs}$ enlarges to $8-12 \mathrm{~mm}$ in diameter that eventually vesiculates and ruptures to form an eschar. ${ }^{1,2}$ It is seen in up to $95 \%$ of cases, is usually painless and single, though occasionally pruritic. $^{2}$ Tender lymphadenopathy may be seen in the draining basin. The eschar resolves in 3-4 wks with no sequalae, but may occasionally cause scarring or hyperpigmentation. ${ }^{1,2}$ As eschar at the bite site is the single most useful diagnostic clue, all febrile patients without localizing signs of infection should be examined thoroughly for the same.

Sivabalan So and Sendil Kumar D Department of Pediatrics, Sundaram Medical Foundation, Chennai - 600040, India. E-mail: sivabalan.somu@gmail.com [DOI-10.1007/s12098-010-0157-3]

\section{REFERENCES}

1. Jeong YJ, Kim S, Wook YD, Lee JW, Kim KI, Lee SH. Scrub Typhus: Clinical, Pathologic, and Imaging Findings. Radiograph 2007; 27: 161-172.

2. Saini R, Pui JC, Burgin S. Rickettsialpox: report of three cases and a review. J Am Acad Dermatol 2004; 51: S137-S142. 